towards the base, healthy ; evidently fatty, competent during life. Aortic orifice enlarged; aortic valves thickened, corrugrated, and calcified throughout; evidently wholly incompetent during life. Ascending aorta greatly dilated, atheromatous, and studded with calcareous matter. Transverse portion of arch the seat of a large aneurism. The sac projected from the anterior superior portion of the wall, and was attached to the upper portion of the sternum, the left clavicle, and first left rib, and pressed upon the trachea and csophagus. It was full three inches in interior diameter; its walls were soft and flabby, about the thickness of normal left auricular parietes. The surface of the sternum, against which the tumor pressed, was eroded, and about half its thickness removed; also, there was erosion of the sternal end of the left clavicle and first left rib. A mass of laminated fibrine the size of a walnut occupied the distal portion of the sac and projected into the ascending aorta. The latter was also much dilated. The innominate and first part of the right subclavian were dilated to about twice the normal diameters. The right and left common carotids and left subclavian were about normal size. Both auricles, the right ventricle, and right-side valves were normal.

This case was complete, and a typical one of combined aortic aneurism and aortic insufficiency. 'The sphygmographic records were also complete. The latter we examine with interest, especially at present, with reference to the succession of the arterial pulses on the systole of the ventricle. Evidently aneurism, as in the present case, of the transverse portion of the arch, involving the origins of the three great arteries, namely, innominate, left common carotid, and left subclavian, entails the same effect on the succession of the pulses as aneurism of the ascending portion only; that is, the pulses are all abnormally delayed, and the symmetrical pulses of either side are equally delayed.

The tracings were taken and the successions measured with the greatest care; so the intervals expressed on the plates are reliable, and must approximate throughout very closely the true ones. The showings are as follows :-

November 28, 1878. No. 79. Pulse-rate 66-60. Cardio-subclavian interval .109 second (average).

No. 80. Pulse-rate 52-51. Cardio-carotid interval .112 second.

December 21, 1879. No. 83. Pulse-rate 82-80. Cardio-carotid interval .0833 second.

No. 84. Pulse-rate 82-80. Cardio-radial interval .1666 second. January 7,1880 . No. 85. Pulse-rate 87-80. Cardio-subclavian interval .0833 second.

These measurements are sufficiently near the normal. In the variations of the time-differences, however, I noticed a bearing towards the longer time, and the average of all the observations, of which a portion only are shown, would indicate rather longer time than obtains in strictly normal conditions, but not sufficiently prolonged to exceed the healthy range or permit the counting of such delay as a definite indication. Then in this case the coexisting aortic aneurism and aortic insufficiency so counterbalanced each other that the retardation of the pulses on the heart was within the normal range.

According to the size and yielding nature of the aneurism must be the amount of delay impressed upon the pulse. An aneurism may be such in these respects as to cause a delay that would overbalance the precipitation entailed by the aortic insufficiency. On the other hand, the conditions may be reversed, and a large aortic insufficiency entail a precipitation that would overbalance the delay caused by the aortic aneurism. However, when the two conditions coexist, the delay will never be so great as to definitely favor the wrong diagnosis of aortic aneurism alone, nor so small as to definitely favor the wrong diagnosis of aortic insuffciency alone. If the diagnosis of aortic aneurism be certain, and the pulse, notably the carotid or subclavian, shows no abnormal delay, the conclusion is justified that aortic insufficiency coexists. If the diagnosis of large aortic insufficiency be certain, and the pulse, notably the carotid or subclavian, shows no abnormal precipitation, the conclusion is positive that aortic aneurism coexists.

So in our case the ordinary signs and symptoms, at the first examination, were sufficient to determine the presence of large aortic insufficiency. Accepting this, the want of abnormul precipitation of the carotid and subclavian pulses enabled me to arrive at the diagnosis of coexisting aortic aneurism before there were any other indications of this condition.

As of interest relating to the localization of aortic aneurism: No. 82 shows the two radial pulses simultaneous, which proves that the aneurism was seated in the ascending portion anterior to the origin of the innominate, or that it was seated in the transverse portion so as to include the origins of both the innominate and left subclavian (and of course also the left common carotid). No 87 shows the left radial as compared with the right femoral slightly delayed. This is the more usual normal relationship of these pulses, and proves that the aneurism was anterior to the origin of the left subclavian artery. Evidently, had the aneurism been below the origin of the left subclavian, the femoral would have shown delay as compared with the radial. No. 86 shows that the pulsation in the second left interspace succeeded the cardiac pulsation about .0714 of a second, and therefore aided the diagnosis of aneurism as located.

No. 80 is given to show a very ample and finely delineated radial pulse, and the rate of transmission of the pulse-wave between the subclavian and radial points under the conditions then existing in the case. The time-difference measures .108 second, which indicates a rather slow pulse-wave transmission. If this be compared with the carotid-radial interval in the case of Quinn, namely, .052 second, as shown in No. 78 , a remarkable difference becomes apparent in the pulse-wave velocity of the two cases. The causes of this diversity are to be found in the dissimilar conditions which pertain to the respective cases.

A CASE OF ENCYSTED ASCITES SIMULATING OVARIAN DRUPSY; OPERATION ; DEATH AU'YOPSY.

BY AUG. F. ERICH, M. D.,

Professor of Diseases of Women, College of Physicians and Surgeons, Baltimore, Surgeon to the Maryland Woman's Hospital.

THe following case, illustrating the difficulties of the differential diagnosis between encysted dropsy of the peritonæum and of the ovary, is published as a contribution to the statistics of those mistakes in diagnosis which to a wise physician are said to be " more instructive than twenty successes." 1

Annie 'Talbott, colored, aged twenty, unmarried, but

1 D. Hayes Agnew. Address before the Pennsylvania State Medical Society, 1878. 
mother of two children, consulted me at the Central Dispensary on August 26, 1875 , on account of an abdominal tumor. Her last child was born in 1872. She has had no abortions. Menstruation began when she was thirteen years old, and continued regularly until between two and three years ago. At that time she began to have pains in the abdomen, and about two years ago she noticed a tumor in the right iliac region. Since then she has had a great deal of pain in that situation, and the tumor has been constantly enlarging. From the first appearance of the tumor her menstruation had become very irregular, and the amount of the discharge had generally been large. During the last six months she had ceased to menstruate. She has never had leucorrhœa. Has much pain while walking, which she describes as sharp and lancinating.

The ahdomen at the umbilicus measured thirty-two inches, the right half being decidedly larger than the left. There were present all the physical signs of a large ovarian cyst in the right side of the abdomen, such as rotundity of the abdomen in the supine posture. dullness on percussion over the surface of the $a b$ domen in the same position, percussion sounds unaltered upon change of position. No evidences of renal, hepatic, or cardiac disease. Skin normal as to color, moisture, etc. No œdema of the feet. The sitting posture affected the shape of the abdomen but little; the line of dullness on its upper boundary, however, was curved, with its convexity upward. Aortic pulsation was transmitted. The uterus was retroverted, of normal size, and slightly movable. A smooth and round tumor occupied the entire roof of the vagina. Her health had not failed more than is usual in ovarian dropsy.

The late Professor Thomas R. Brown, whose untimely death leaves a void in the ranks of progressive surgeons not easy to fill, was present at the examination, and stated that the patient had been under his care for some time; that he had examined her carefully, and diagnosticated unilocular ovarian cyst. $\mathrm{He}$ had evacuated the cyst by tapping, and finding, upon careful palpation of the abdomen, only so much of the cyst remaining as could be attributed to the collapsed cyst walls, and the fluid evacuated corresponding to all the chemical and microscopic characters stated to be present in ovarian cystic fluids, he sent the case to me for operation. Concurring in the opinion of Professor Brown, with reference to the nature of the case, I determined to operate as soon as more favorable weather set in.

The patient, having suffered much from pain and loss of sleep, presented that peculiarly anxious expression of countenance so characteristic of ovarian dropsy. She was placed upon tonics and alteratives, and also opiates for the relief of pain and sleeplessness. Under this treatment she soon gained flesh, and lost entirely that anxious expression of the face already mentioned, so that on the day of operation she presented a good supply of adipose tissue, and was otherwise in good condition for the operation.

About the middle of September the patient had an attack of peritonitis, which, however, yielded readily to large doses of morphia and quinine. A few days before the operation she was etherized and subjected to another most careful examination, in the presence and with the assistance of Professors Brown and Lynch. During this examination two additional facts were elicited :-
(1.) The presence of a small amount of ascitic fluid in the peritoneal cavity, especially on the left side of the abdomen.

(2.) Slight resonance at a circumscribed point upon the anterior surface of the tumor, near the umbilicus.

The small amount of the,ascitic fluid was thought to be the result of the recent attack of peritonitis, and the circumscribed resonance attributed to a loop of intestine adhering to the anterior surface of the tumor, a rare condition referred to by Peaslee. ${ }^{1}$

No other disease except encysted dropsy of the peritonæum could present so many characteristic signs of ovarian cyst as were present in this case; but so many of the supposed distinctive signs of this disease, as laid down by Peaslee, ${ }^{2}$ were absent, that the diagnosis of ovarian cyst was, I think, justified. The diagnostic features of ovarian dropsy, as given by Peaslee, were nearly all present, as rapid growth of the tumor, impairment of health, with the peculiar features, prominence of abdomen, tumor felt per vaginam, uterus behind tumor, large quantity of fluid, und, as positively stated by Professor Brown, a microscopist of experience, the presence of the so-called "ovarian cell." In view of these facts it was decided to operate. On October 29, 1875, after all the usual preparations for ovariotomy had been made, the patient was etherized, and an explorative incision of three inches in length was made in the linea alba. Upon entering the peritonæum a profuse quantity of amber-colored fluid gushed forth, making it evident that the cyst had been opened. An introduction of the hand into the cavity soon satisfied me that the case was one of encysted ascites. I next proceeded to detach the adhesions, and in doing so opened the main cavity of the peritonæum, which was indicated by a new gush of a similar fluid. Finding what seemed to be small cysts between coils of intestine adherent to each other, I enlarged the incision to five inches, opened a number of cysts ranging in size from a hen's egg to an orange, and after carefully cleaning out the abdominal cavity a Thomas's drainage tube was introduced, and the wound closed by deep sutures of silver wire and superficial sutures of silk. 'The time occupied in the operation from the first incision to the closing of the wound was sixty-one minutes.

Immediately after the operation the patient's temperature was $98.20^{\circ} \mathrm{F}$.; pulse 100 . She was put to bed, and pain averted by hypodermic injections of morphia. She was nourished by rectal injections of a mixture consisting of beef essence, sweet cream, egg, and whisky. In addition four grains of quinine were given, also per rectun. Later, when the injections were no longer retained, astringents were added to check the diarrhœe. Nothing but ice with a little whisky or table tea could be given by the mouth without exciting vomiting. The abdominal cavity was washed out every eight hours with carbolized water of the strength of sixteen grammes to the pint. Later, one drachm of chloride of sodium was added to each pint of the injection. The temperature, pulse, and respiration were recorded every six hours. 'The temperature generally ranged between $100^{\circ} \mathrm{F}$. and $101^{\circ} \mathrm{F}$, only four out of the observations taken rising as ligh as $103^{\circ} \mathrm{F}$. The range of the pulse was between 140 and 160 , and the respiration between 20 and 24 . The observation taken 1 Ovarian Tumors, New York, 1872, page 150.
2 Op. cit., pages $155,156$. 
on the sixth day after the operation and five hours before death was, temperature $101.5^{\circ} \mathrm{F}$.; pulse 140 , respiration 18 .

Symptoms of septicæmia were developed as early as the third day. Neither temperature, pulse, or respiration indicated the approach of death. The rapidly failing strength and progressively increasing loss of consciousness were the only indications of that issue. Death took place at 1.53 P. M., of November 4 th, the patient having lived a little over six days after the operation. The autopsy was held by my colleague Professor Bevan, whose report is as follows :-

POST-MORTEM EXAMination OF ANNIE talbotT.

"Section made eight hours after death, November 4, 1875 : -

"'The wound through the abdominal walls made at the operation was five inches in length; the lower part of this incision externally had united around the drainage tube; the external upper part of wound ununited. At poiuts corresponding to the wire and silk sutures adhesion had taken place.

"The post-mortem incision was made from the median line to the angle of the eighth rib, then to crest of the right ilium, along Poupart's ligament to the crest of the left ilium, and the flap thus included turned to the left side.

"The ascending and transverse colon was adherent to the parietes; the stomach was bound firmly to the liver and transverse colon. The peritonæum, both visceral and parietal layers, was of a creamy white color, and everywhere covered with recently exuded lymph. The whole mass of the intestines was firmly bound together, to the neighboring organs and to the abdominal walls. Here and there small pouches or cysts, containing serum, and varying in size from a walnut to a cocoanut, were found. They were completely enveloped by either peritoneal adhesions or bridges of lymph. Immediately under the liver and between that organ and the ascending colon was quite a large pouch or cyst; another cyst was found on a line with the umbilicus, between the internal border of the colon, small intestines, and abdominal parietes. The entire contents of the abdomen were bathed with a yellowish ochre-colored fluid of a highly offensive odor. The drainage tube had passed behind the uterus, Jymph had formed a complete wall around it, and by an adhesion between the posterior and lower portion of the uterus and the rectum Douglas's cul-de-sac had been obliterated. The ovaries were normal. A cyst of the size of a hen's egg was found at the fundus uteri. The peritonæum over and around the symphysis pubis was much thickened and easily torn. The internal surface of the operative incision was united at one or two points, whilst its edges generally were blackened and gangrenous; this gangrenous condition extended some distance on the small intestines beneath the wound. Kidneys normal. Liver of normal size and color, but very friable. Lungs adherent by their bases to the pleuræ and diaphragm, but otherwise natural. No evidences of tubercle or glandular enlargement about the body. Heart normal."

The death of the patient was evidently due more to septicæmia than to peritonitis. Perfect drainage and washing out of the abdominal cavity was rendered impossible by the numerous divisions formed by the adhesions between the intestines. In addition, the intestines surrounding the drainage tube had become? agglutinated, thus preventing the water entering the main cavity of the peritonæum. This accident suggests the propriety of connecting the drainage tube with the injection apparatus in such a manner as to cause the water partially to fill and distend the cavity before allowing it to escape. I have learned to consider a drainage tube a sometimes necessary evil, and think that it should be dispensed with whenever the peritonæum can be kept clean without it.

An examination of the fluid removed from the cysts gave a specitic gravity of 1030 ; it was of an amber color, did not coagulate spontaneously, and left a thick deposit. A microscopical examination discovered granular bodies corresponding to the "ovarian cell "described by Dr. Drysdale, of Philadelphia, and by him considered diagnostic of ovarian cysts.

After a careful consideration of all the circumstances of this case, and after no inconsiderable subsequent experience, I am as yet "aware of no means by which such cases are to be distinguished from ovarian dropsy." 1

\section{RECENT PROGRESS IN ANATOMY.}

BY THOMAS DWIGHT, M. D.

\section{THE DEVELOPMENT OF THE STERNUM AND OF THE STERNO-CLAVICULAR JOINT. ${ }^{2}$}

THE observations recorded in this paper refer to three points in the early development of the human sternum and its neighboring parts, namely: the bone itself, the ensiform cartilage, and the parts included between the inner ends of the clavicles. The work has been carefully performed, and the article is about as readable as the nature of the subject will permit. Dr. Ruge's method has been to examine the parts of young human embryos in glycerine with a low power, and then to make a number of microscopic sections of them.

Two views have been held as to the development of the sternum; one that it is formed by the fusion of two lateral halves, the other, that it is originally a median organ. The latter view, which, we believe, has had but few supporters, may now be definitely set aside, at least for the human sternum. Dr. Ruge unfortunately has not seen any of the very earliest stages, but he shows that the two halves appear in connection with the ribs. In a foetus three cm. long they are still separate, though nearly touching in the region of the manubrium. It is there that they first unite, and the line of union runs downward. A transverse line between the second ribs shows the separation of the manubrium from the body. In early stages a similar line is observed between the third ribs. This subsequently disappears in a way that recalls the primary segmentation of the notochord.

Dr. Ruge finds that the ensiform cartilage is also double in its origin, and is formed in a similar way in connection with the ends of the eighth and ninth ribs. A retrograde metamorphosis on each side of this cartilage brings about the adult condition. The author calls attention to the fact that most mammals, and even some of the highest, have more than seven pairs of sternal ribs; hence their transient appearance in the embryo is in accord with several well known phenomena. The occasional variations observed in the

1 T. Spencer Wells. Disease of the Oraries, page 134. 1880 .

By G. Ruge. Morphologisches Jahrbuch, Band VI., Heft 8 